\title{
Ruta histórica cultural “ El Abra " un producto turístico enfocado al turismo interno en la isla de la juventud
}

\section{Historical cultural route "El Abra" a tourist product focused on domestic tourism on the isla de la juventud}

Leilyn Arias Pérez. ${ }^{1}$, Wenceslao González Curbelo. ${ }^{2}$ \& Lourdes Cisneros Mustelier. ${ }^{3}$

\begin{abstract}
.
Historic-cultural tourism has become an option that allows the interpretation, conservation, presentation and increasing importance of the assets that relate history and cultural heritage, hence the importance of designing tourism offers that guarantee the relationship between community, visitors and heritage in a sustainable environment. The present investigation was proposed as a general objective to design the historical - cultural Route "El Abra" that contributes to the consolidation of internal tourism (in popular slang it is known as the national market) in the Isle of Youth; making possible the use of natural and historical cultural resources existing in the area, relying on the tourism development objectives of the country and the growing demand of this tourist modality. The selection of the place was based on the excellent conditions it has for the practice of sociocultural and natural tourism, distinguished by its high historical value patrimonial and the existence of important natural resources. Based on the proposed objectives, several procedures for the design of tourism products were reviewed, selecting the procedure proposed by Perelló (2001). The process is aligned with the guidelines outlined by the Seventh Congress and the Guidelines for Economic and Social Policy of the Communist Party of Cuba, approved by the National Assembly of People's Power in July 2016.
\end{abstract}

\footnotetext{
${ }^{1}$ Centro de Capacitación del Turismo, Isla de la Juventud, La Habana, email leilyn@iju.mintur.gob.cu

${ }^{2}$ Centro de Capacitación del Turismo, Isla de la Juventud, La Habana, Cuba, email gwence@iju.mintur.gob.cu

${ }^{3}$ Facultad de Turismo-Universidad de La Haban, Cuba, email lourdes_cisneros@ftur.uh.cu
} 
Keywords internal tourism, historical - cultural route, local development.

\begin{abstract}
Resumen.
El turismo histórico-cultural se ha convertido en una opción que permite la interpretación, conservación, presentación y puesta en valor de los bienes que vinculan la historia y el patrimonio cultural, de ahí la importancia del diseño de ofertas turísticas que garanticen la relación entre la comunidad, los visitantes y el patrimonio en un entorno sostenible. La presente investigación se planteó como objetivo general diseñar la Ruta histórica - cultural "El Abra" que contribuya a la consolidación del turismo interno (conocido en el argot popular como mercado nacional) en la Isla de la Juventud; posibilitando el aprovechamiento de los recursos naturales e histórico - culturales existentes en el área, apoyándose en los objetivos de desarrollo turístico del país y en la demanda creciente de esta modalidad turística. La selección del lugar estuvo fundamentada por las excelentes condiciones que posee para la práctica del turismo sociocultural y natural, distinguiéndose por su elevado valor histórico - patrimonial y la existencia de importantes recursos naturales. En función de los objetivos propuestos fueron revisados varios procedimientos para el diseño de productos turísticos, seleccionándose el procedimiento propuesto por Perelló (2001). El proceso se encuentra alineado con las pautas trazadas por el VII Congreso y los Lineamientos de la Política Económica y Social del Partido Comunista de Cuba, aprobados por la Asamblea Nacional del Poder Popular en julio de 2016.
\end{abstract}

Palabras clave turismo interno, ruta histórico - cultural, desarrollo local.

\title{
Introducción.
}

El turismo es por su naturaleza un fenómeno psicosocial que no puede desvincularse de su condición individual y colectiva, es decir, no es posible separar ni de la persona, ni de la estructura social y por tal motivo precisa de un enfoque transdisciplinario. En esta misma dirección, el turismo hace muchos años se definió no como una corriente de personas, ni como una expedición o viaje, sino como un fenómeno social vinculado a las relaciones interpersonales y a la comunicación humana.

Sin embargo, la compleja situación internacional, tanto económica como de riesgos bélicos y epidémicos, ha provocado la desaceleración de los flujos de viajeros desde los llamados mercados de larga distancia. Para Cuba, la opción de estabilizar el mercado nacional como segmento turístico, puede representar una ventaja para disminuir la marcada estacionalidad y aumentar las tasas de ocupación hotelera (Perelló, 2015).

Según la Organización Mundial del Turismo (OMT), el turismo comprende las actividades que realizan las personas durante sus viajes y estancias en lugares distintos al de su entorno habitual, por un período de tiempo consecutivo inferior a un año, con fines 
de ocio, por negocios y otros motivos. Serán considerados turistas, los visitantes que pernocten al menos una noche en el lugar visitado en un alojamiento colectivo o privado. Se incluyen aquí a los no residentes extranjeros, a los nacionales residentes fuera y dentro del país, entre otros.

La nomenclatura de la OMT sostiene que, el turismo interno es el que concierne a los residentes del país de referencia, cuando se encuentran de viaje dentro de su país. Además, se define como turismo nacional a los viajes de los residentes dentro del territorio de su país, así como al exterior.

El turismo interno, dentro del Sistema de Turismo, representa un importante segmento de consumidores, no solo para el hotelería, sino también para las entidades asociadas a la venta de paquetes de excursión, renta de medios de transporte, restauración y venta de productos como los de la red de tiendas Caracol.

En los lineamientos aprobados para el turismo, en el VI y VII Congresos del Partido Comunista de Cuba (PCC) se plantea respectivamente: "Dinamizar e impulsar el desarrollo del turismo nacional mediante la creación de ofertas que posibiliten el mayor aprovechamiento de la infraestructura creada en hoteles y otros atractivos turísticos recreativos e históricos. Estudiar una política que facilite a los cubanos residentes en el país viajar al exterior como turistas" (PCC, 2011:34) y consolidar el turismo interno, creando y diversificando ofertas que posibiliten el mayor aprovechamiento de las infraestructuras, así como otras ofertas que faciliten a los cubanos residentes en el país, viajar al exterior como turistas (PCC, 2016:214).

En el país se ha notado el incremento de los visitantes internos, sin embargo, en la Isla de la Juventud esta cifra aún es baja. En los años comprendidos del 2011 al 2016 visitaron el territorio pinero 126828 cubanos, oscilando los niveles de ocupación en la red de hotelería local que opera el turismo interno entre los 16893 y 24802 nacionales anualmente, según datos ofrecidos por la Oficina Nacional de Estadísticas e Información.

Diseñar una ruta turística para potenciar el crecimiento del segmento turístico de los cubanos residentes como consumidores turísticos, resulta un imperativo en las actuales condiciones de desarrollo de la economía nacional en general y del sector turístico en particular, sobre todo en una zona que además recibe poco turismo internacional, como lo es la Isla de la Juventud.

De todo lo anterior se define como problema de la investigación, ¿cuál será la incidencia de las propuestas de nuevos espacios u ofertas turísticas en la Isla de la Juventud para el turismo interno? El objetivo general es Diseñar la Ruta histórica - cultural "El Abra" que contribuya a la consolidación del turismo interno en la Isla de la Juventud. Como objetivos específicos se plantearon los siguientes: 
1. Describir el marco teórico del turismo a partir de sus conceptualizaciones y su situación en Cuba bajo las condiciones del actual entorno económico.

2. Caracterizar las potencialidades que tiene la Ruta histórica - cultural "El Abra" como producto turístico enfocado al turismo interno.

3. Determinar los elementos conformadores de la propuesta a realizar.

La presente investigación resulta de marcada importancia para la localidad, la región y el país en general pues de este modo se pueden atraer nuevos clientes del turismo interno, estableciendo las prioridades que posibiliten un desarrollo sustentable y permitan al visitante percibir los encantos patrimoniales y naturales que atesora la Isla joven. Razón por la cual se considera que la propuesta de diseño de la Ruta histórica - cultural "El Abra" puede insertarse como una vía para a dar a conocer y poner en valor la historia, naturaleza y patrimonio que atesora el territorio pinero, convirtiéndose en una herramienta de gestión del turismo en la localidad y de esta manera contribuir al desarrollo regional.

\section{Métodos.}

La investigación presenta una perspectiva metodológica cualitativa, tomando en consideración que la misma se basa en un proceso hipotético - deductivo, donde se analizan, interpretan y describen informaciones o realidades de hechos en su entorno natural; en aras de generar, posteriormente, aspectos teóricos en cuanto al fenómeno analizado y alcanzar su correcta interpretación. Los principales métodos científicos en los que se apoya son:

Analítico - sintético: se empleó en la búsqueda de las relaciones entre los diferentes aspectos conceptuales de la investigación, así como la generalización a partir de la información obtenida en los distintos instrumentos aplicados, en franca correspondencia con los objetivos propuestos. Mediante la utilización del mismo fue posible analizar y sintetizar los resultados obtenidos de las entrevistas a especialistas, contribuyendo al arribo de las conclusiones.

Hipotético - deductivo: La investigación parte de una hipótesis que se sustenta en una base teórica. Es posible llegar a conclusiones a partir de la hipótesis mediante la deducción, que después se pueden comprobar: a partir de la oportunidad que representa el diseño de una ruta histórica - cultural se puede inferir la puesta en valor de la Finca Museo "El Abra” y el Complejo Recreativo de igual denominación.

Histórico - lógico: Se halla vinculado con el conocimiento de las distintas etapas de los objetos en su sucesión cronológica. Se analizó cómo existe una evolución en la historia de la Finca - Museo "El Abra" y el Complejo Recreativo de igual nombre, así como en los conceptos turismo, recursos naturales e histórico - culturales, diseño de producto 
turístico y su relación con las conexiones históricas fundamentales; amén de posibilitar la aproximación al comportamiento del campo de acción en el diseño de la propuesta, de lo simple a lo complejo, según los grados de desarrollo del conocimiento. Se utilizaron además métodos empíricos como:

El análisis de documentos: permitió profundizar y ampliar en las diferentes bibliografías la información y conocimientos precisos sobre el objeto de investigación y de esta forma confeccionar el marco teórico de una forma crítica.

Observación Participativa: se realizó para obtener información directa de los objetos estudiados en sus condiciones naturales. El empleo de este método permitió tener una percepción objetiva respecto a las actividades desarrolladas en la Finca - Museo "El Abra" y en el Complejo Recreativo de igual denominación. Atendiendo a los medios empleados se clasifica en estructurada o sistémica, pues se utilizaron como medios técnicos el teléfono móvil para grabar y fotografiar, así como la libreta de anotaciones - de vital importancia - pues coincidiendo con Sampieri y otros (2014): "las anotaciones evitan que se nos olviden aspectos que observamos; no escribirlas es como no observar". Atendiendo al lugar donde se efectuó se clasifica en real o natural puesto que se realizó en el lugar del objeto investigado.

Entrevista: se utilizó con el objetivo de obtener la información confiable, necesaria para desarrollar la investigación de manera vasta y directa. También como vía para la validación de la ruta histórica - cultural por los recursos naturales e histórico - culturales de la Finca - Museo "El Abra” y el Complejo Recreativo de igual denominación. Estuvo dirigida a especialistas en diversos temas, tales como historia, turismo y patrimonio, entre otros, con el fin de obtener su opinión sobre los principales elementos o recursos que se podrían incluir en el recorrido, las características de estos recursos, su importancia a lo largo de la historia, los valores arquitectónicos, culturales y patrimoniales, que a su consideración, los hacen merecedores de su selección y al mismo tiempo retroalimentar los resultados obtenidos del análisis documental y la observación científica.

Encuestas: Este método permitió conocer las necesidades y expectativas actuales del turismo interno, además de su percepción y el estado de opinión acerca la creación de nuevas ofertas de productos turísticos que integren componentes naturales e histórico culturales en la Isla de la Juventud.

Estadístico - matemático: La técnica que se utilizó para el análisis de los resultados fue la tabulación simple, mediante el empleo del programa Microsoft Excel como eficaz herramienta informática en el procesamiento de datos. La información obtenida de las encuestas se presentó en gráficos, viabilizando así el análisis de la información.

Durante la realización de la investigación se consultaron varias fuentes, primarias y secundarias, en distintos tipos de soportes. 
Fuentes de información primaria: se obtuvo mediante la aplicación de técnicas de obtención de información primaria como son la observación científica, la realización de entrevistas, la aplicación de encuestas a clientes del turismo interno, así como el análisis general de la Finca - Museo y el Complejo Recreativo "El Abra".

Fuentes de información secundaria: provino principalmente de documentos y de datos históricos de la Finca - Museo y el Complejo Recreativo "El Abra" (se utilizó esencialmente para la caracterización de ambas instalaciones); de publicaciones y sitios web nacionales e internacionales, de Tesis de Diploma, de Maestrías y Doctorales realizadas anteriormente en el país, entre otras. Aunque estas fueron de vital importancia para todo el estudio se utilizaron fundamentalmente para la conformación del marco teórico - conceptual.

Para el diseño de la ruta se utilizó el esquema metodológico propuesto por Perelló, (2018). En tal sentido, se tuvo en cuenta dentro de este esquema la Fase I, referente a la Demanda, en la que se determinó como principal cliente al turismo interno, debido a la necesidad creciente de conocer las características e historia que atesoran la Finca Museo y el Complejo Recreativo "El Abra", además del interés de la localidad por la divulgación de estos conocimientos que forman parte del proceso histórico - revolucionario cubano y de la identidad del pueblo pinero. La ruta se encuentra abierta además a visitantes de otras nacionalidades que deseen conocer y comprender los elementos comprendidos en la misma. Posteriormente se pasó a la Fase II, relacionada con el Diseño del Producto, donde se tomó en cuenta la Primera Etapa relativa a los recursos y atractivos. Se determinó que en el lugar donde se desarrollará la ruta existen recursos naturales y patrimoniales de considerable valor que atraerán la atención de los visitantes. Luego se transitó a la Segunda Etapa en la que se plantea la generación de ideas, donde se comenzó a esbozar cómo podría realizarse una ruta que incluyera la Finca - Museo "El Abra" y el Complejo Recreativo de igual denominación. Finalmente se definieron las actividades propias que se incluirían dentro de la ruta y las acciones generales que deberán llevarse a vías de hecho en aras de alcanzar el óptimo desarrollo del referido producto. La conformación de la propuesta se desarrolló hasta este momento, razón por lo cual no se reflejó en el trabajo de investigación el resto de la metodología.

\section{Resultados.}

Derivado de la utilización de los métodos y las técnicas aplicadas durante el transcurso de la investigación se obtuvieron resultados significativos que aportaron validez a la propuesta del diseño de la ruta, a saber:

Observación participativa: la observación realizada permitió comprobar la información que se tenía sobre las condiciones de los recursos turísticos naturales y antrópicos con los que cuenta la zona comprendida en la Ruta histórica - cultural "El Abra". Resultando relevante destacar que, desde el punto de vista de conservación, la mayoría de los recursos 
tanto naturales como antrópicos se encuentran en buenas condiciones y con grandes posibilidades para ser explotados turísticamente por el mercado nacional. Sin embargo, se precisa la realización de varias acciones para la optimización del desarrollo del producto.

En cuanto a la protección y conservación de las condiciones constructivas se constató la reciente remodelación del museo en cumplimiento de la primera etapa del Proyecto de Rescate para el Monumento Nacional Finca "El Abra"; así como el inicio de la segunda etapa consistente la reparación de las 17 viviendas localizadas en el lugar, la rehabilitación del manantial y el horno de cal, acondicionamiento del camino que conduce de la finca al manantial, recuperación del bosque martiano y restauración del portón que da acceso a la finca. Respecto a las instalaciones ubicadas en el Complejo Recreativo su mayoría se encuentran en buenas condiciones técnico - constructivas, aunque resulta necesario remozar algunas áreas (parque infantil, puente situado en el embalse, señales que brindan información sobre los sitios adecuados para bañarse en la presa, sombrillas de madera y guano ubicadas en la orilla del embalse) y restaurar otras (parqueo, el mirador y sus instalaciones anexas, barandas de la escalera mediante la cual se asciende a este lugar). Se observó que la carretera que conduce a los sitios comprendidos en la ruta se encuentra en buen estado. No obstante, se apreció el deficiente estado de conservación de la valla que brinda información sobre la localización de la Finca - Museo, así como la ausencia de señalización que oriente la localización del Complejo Recreativo "El Abra". Se verificó que, dado el carácter patrimonial del lugar, todas las acciones de rehabilitación realizadas en la Finca - Museo se basan en el absoluto respeto por el entorno natural y los inmuebles situados en la zona; proceder compartido además por trabajadores, residentes y visitantes.

Se comprobó, respecto al Complejo Recreativo que, aunque mantiene su conexión al Sistema Electro - Energético Nacional, las principales fuentes de energía utilizadas para la iluminación y cocción de alimentos son: la solar, la leña y el carbón; todo lo cual, como parte también de su vocación de sostenibilidad, se halla a la vista del visitante. Amén de constatarse que los residuales líquidos se evacúan hacia una planta compacta para su tratamiento, cuya disposición final, en forma sólida y líquida es reincorporada sanitariamente al medio. Los residuales sólidos son clasificados y depositados de manera transitoria en puntos sanitarios apropiados para su posterior entrega a los órganos encargados de su colecta y reciclaje.

Los recursos humanos que laboran en la Finca - Museo "El Abra" pueden catalogarse como homogéneos, al encontrarse conformado el colectivo por técnicos medio egresados de los centros de Instructores de Arte, fundamentalmente y trabajadores de nivel superior, graduados de Especialidades en la rama de Museología. Situación similar se constató en el Complejo Recreativo donde todos los empleados son egresados de nivel medio superior o superior en las distintas especialidades de comercio, gastronomía o economía, en consonancia con las plazas que ocupan. 
Se observó que la profesionalidad y la instrucción de los trabajadores, en ambos sitios, resultan adecuadas, en correspondencia con la labor que desempeñan.

En sentido general, a partir de la observación realizada, se constató que el perímetro escogido para la implementación del producto posee las características ideales para el desarrollo de la Ruta histórica - cultural "El Abra".

Entrevistas: para la valoración de la propuesta se seleccionaron varios especialistas (tres directivos, cuatro funcionarios y dos profesores) tomando en consideración determinados criterios basados en la experiencia que tienen en el tema, relacionados con el turismo, diseño de productos turísticos, historia y patrimonio; valorando además que se encuentran vinculados a la zona objeto de estudio ya sea por conocimientos, como por los centros en los cuales laboran (pertenecientes al Ministerio de Turismo y al de Cultura). Se les realizó una ronda de preguntas a fin de obtener la mayor información posible, así como sus consideraciones personales.

\section{Los resultados de este método se resumen en los siguientes aspectos:}

- El $100 \%$ de los entrevistados afirmó que el desarrollo de nuevos productos que integren componentes naturales e histórico - culturales podrían contribuir al impulso del turismo interno en la Isla de la Juventud, coincidiendo en que el territorio posee potencialidades para el desarrollo de productos turísticos que integren componentes naturales e histórico - culturales. El 88,9\% se refirió a los beneficios que podría ello reportar a la localidad, entre los cuales citaron: el necesario desarrollo de infraestructuras, dinamización de las inversiones, generación de empleos tanto en la actividad turística como en otras ramas vinculadas a ellas, estimulación de la continuidad histórica de la comunidad pinera, contribución a la rehabilitación y conservación de monumentos y sitios históricos, revitalización de costumbres y mejoramiento de la sanidad ambiental; en tanto el 11,1\% mostró preocupación acerca de los valores naturales, históricos y culturales que pudieran resultar afectados por el desarrollo de la actividad turística.

- El 100\% de los entrevistados aseveró que la Finca - Museo "El Abra" y el Complejo Recreativo de igual denominación cuentan con suficientes recursos o atractivos de carácter natural e histórico - cultural que pudieran ser utilizados para el desarrollo de algún producto, a la vez que reconocían las grandes potencialidades de la zona en que se hallan ubicadas ambas instalaciones para su implementación. El 88,9\% afirmó que el desarrollo de la actividad turística resultaría beneficioso para la comunidad local, provocando un cambio positivo aunque gradual - respecto a la imagen debilitada que proyecta actualmente la Isla de Juventud como destino, a la vez que admitieron los impactos positivos que implicaría el desarrollo del turismo en el territorio; el otro 11,1\% no discrepó de 
estas opiniones pero también hizo referencia a los posibles impactos negativos que pudiese ocasionar la intervención continua de personas en las áreas concebidas para la explotación de los productos turísticos diseñados al efecto.

- De igual modo, el $100 \%$ de los entrevistados se mostraron conformes con el diseño de una ruta histórica - cultural para el turismo interno, que comprenda la visita a la Finca - Museo "El Abra” y el Complejo Recreativo de igual denominación. Reconocieron además la escasez de ofertas de este tipo en la localidad y la consiguiente insatisfacción del turismo interno respecto a la misma. Razones por las cuales estuvieron de acuerdo con la propuesta.

Durante el transcurso de las entrevistas resultaron significativos variados elementos que aportaron validez a la propuesta del presente trabajo investigativo:

- Progresivo aumento de la tendencia mundial - con repercusión nacional - respecto al consumo de productos turísticos basados en la visita a sitios de interés histórico - natural y patrimonial.

- Elevada aceptación de este tipo de productos por el mercado nacional.

- Reconocimiento de la validez de la propuesta, en correspondencia con los Lineamientos de la Política Económica del país.

- Tanto la Finca - Museo "El Abra" como el Complejo Recreativo de igual nombre poseen suficientes atractivos y recursos para diseñar una ruta histórico - cultural que coadyuve a la puesta en valor, la comprensión histórica - patrimonial y la divulgación de los conocimientos relativos a los elementos comprendidos en la ruta y su entorno.

- Reconocimiento de la necesidad de optimizar la infraestructura turística existente en el territorio en aras de complementar la propuesta, contando los elementos comprendidos en la ruta con valores históricos, culturales y patrimoniales que elevarían el atractivo del producto turístico.

\section{Encuestas: (Muestra de 90 clientes)}

Se aplicó un muestreo intencional que según Sampieri y otros (2014) es utilizado en estudios exploratorios y en investigaciones de tipo cualitativo. Se eligió una cifra de personas considerada como representativa, valorando que los encuestados podían proporcionar la información con la profundidad, riqueza y calidad necesarias.

La realización de las encuestas permitió la identificación de oportunidades para introducir nuevos productos en el territorio pinero, al tiempo que se detectaron aquellas expectativas que aún no han sido satisfechas por las opciones actualmente disponibles. 
Se encuestaron noventa clientes hospedados indistintamente en viviendas de arrendadores de habitaciones del sector privado y en el Hotel La Cubana, perteneciente a la Empresa de Comercio y Gastronomía en el Municipio Especial Isla de la Juventud.

La encuesta fue aplicada durante los meses de marzo y abril del año 2018; meses escogidos con intencionalidad debido a la considerable afluencia de turismo interno que acude al municipio motivados por las fiestas pineras (marzo) y la semana de receso escolar (abril). Mediante la misma se constató que el 100\% de los encuestados hizo referencia a la aceptación que tendría la creación de nuevas ofertas de productos turísticos que integren componentes naturales e histórico - culturales en la zona. Se sometió a su consideración el tipo de producto que les gustaría, advirtiéndose como resultado que el $6,7 \%$ de la muestra prefirió la creación de un producto que consista solo en la visita a la Finca - Museo "El Abra”, el 12,2\% seleccionó un producto que consista solo en la visita al Complejo Recreativo de igual nombre, mientras que el $81,1 \%$ optó por un producto turístico que comprenda la visita a ambos sitios.

Tomando en consideración los resultados teóricos obtenidos durante el proceso investigativo fue posible constatar que tanto la Finca - Museo "El Abra" como el Complejo Recreativo de igual nombre poseen suficientes atractivos y recursos para diseñar una ruta histórica - cultural que coadyuve a la puesta en valor, la comprensión histórica - patrimonial y la divulgación de los conocimientos relativos a los elementos comprendidos en la ruta y su entorno.

\section{Propuesta de la Ruta Histórica - Cultural "El Abra".}

El concepto del producto Ruta histórica - cultural "El Abra" debe brindar experiencias memorables sustentadas en elementos históricos - patrimoniales e identitarios de la comunidad pinera. El desarrollo del producto se concibe en varias etapas, atendiendo al siguiente diseño:

- Diseño del producto: Modalidad a desarrollar Turismo histórico - cultural.

- Nombre comercial del producto: Ruta histórica - cultural "El Abra".

- Producto básico: Desarrollo de experiencias vinculadas con actividades al aire libre mediante paseo por la Finca - Museo "El Abra" y sus alrededores, presentación de material audiovisual referido a la estancia de José Martí en la hacienda, conversatorio con descendientes de la familia Sardá - Valdés para conocer elementos históricos o anécdotas relacionadas con el Apóstol u otras temáticas que resulten de interés para los visitantes; unido al recorrido por el Complejo Recreativo "El Abra", disfrutando de variadas actividades en el embalse, parque infantil, mirador, restaurante, parrilladas y bares existentes en el lugar. 
Necesidad del cliente que satisface: Adquisición de experiencias sobre la historia cubana, así como de peculiaridades relativas a la cultura e idiosincrasia pineras.

- Producto periférico: Remodelación de infraestructura para la optimización de los servicios que se brindarán.

Necesidad del cliente que satisface: transportación, alimentación y adquisición de recuerdos.

- Producto complementario: Se añade valor a partir de los recursos naturales, culturales e históricos que existen en la región, desarrollando recorridos y breves excursiones.

\section{Programa de recorrido para la Ruta histórica - cultural "El Abra".}

La Ruta histórica - cultural "El Abra" comprende un recorrido en ómnibus de pequeño formato, desde el Km. 0, situado en la ciudad de Nueva Gerona, realizando una primera parada en la Finca - Museo "El Abra", continuando posteriormente hacia el Complejo Recreativo de igual denominación. Culmina el recorrido retornando al Km.0 de la ciudad (Anexo 2).

Concepto: Opcional de un día, modalidad de turismo histórico - cultural.

Mercado: Turismo interno, segmento de excursión.

Duración del recorrido: 7 horas con 30 minutos; se requiere asistencia de un guía especializado.

Objetivo del recorrido: Potenciar - mediante visitas nacionales - la divulgación de la historia que atesora la Finca - Museo "El Abra", al tiempo que conocen los visitantes rasgos identitarios de la cultura pinera.

Programa: Inicio del recorrido desde el Km 0. de la ciudad de Nueva Gerona. Arribo a la Finca - Museo "El Abra". Una vez allí, en un espacio especialmente destinado para ello, se brindará a los visitantes coctel de bienvenida, informándoseles sobre el Producto y sus ofertas. Luego se trasladarán en el ómnibus hacia el Complejo Recreativo. Finaliza el recorrido retornando al $\mathrm{Km}$ 0. de la ciudad.

\section{Las ofertas comprendidas en el Programa consisten en:}

- Visita a la Finca - Museo "El Abra".

- Presentación de material audiovisual sobre la estancia de José Martí en la Finca. Compartir con descendientes de la familia Sardá - Valdés: historias, anécdotas. 
- Ligera caminata hasta el valle del manantial por el sendero interpretativo creado al efecto, donde podrán beber de sus aguas, apreciar la belleza de la avifauna y descansar brevemente.

- Traslado en ómnibus hacia el Complejo Recreativo "El Abra".

- En el embalse disfrutar de actividades náuticas consistentes en paseos a bordo de botes, kayaks, catamaranes veleros, bicicletas y motos acuáticas. Baño en zonas debidamente señalizadas.

- Realización de actividades recreativas en el parque infantil.

- Disfrutar la belleza del paisaje desde el mirador situado en la cima una de las elevaciones de Sierra de Casas.

- Disfrutar las ofertas de servicios gastronómicos (almuerzo), coctelería y otras bebidas nacionales.

El estudio realizado permitió diseñar la Ruta histórica - cultural "El Abra" en un agradable nicho temático - motivacional; brindando un aporte a la sociedad, debido a que la puesta en práctica de la misma puede contribuir al afianzamiento de la identidad cultural a través del disfrute y la utilización racional de los diversos atractivos turísticos que la integran. Se espera además que, en un futuro, la presente investigación conlleve a la toma de decisiones por parte de los agentes o de las instituciones con competencia en el tema, en aras de viabilizar los estudios correspondientes que posibiliten su comercialización y puesta en valor.

\section{Conclusiones:}

- El desarrollo del turismo interno (mercado nacional) supone la extensión del modelo turístico del ámbito internacional al ámbito interno, contribuyendo a la expansión de la actividad a diversos territorios del país, en ocasiones poco transitados - aunque conocidos - en su relación histórica.

- En este sentido, el diseño de la Ruta histórica - cultural "El Abra" se inscribe como un producto novedoso, que al mismo tiempo convierte la historia en una oferta colaborativa y solidaria, dirigida al turista interno, empleando ómnibus de pequeño formato, con el acompañamiento de un guía de interpretación convenientemente formado y con elevado conocimiento de la historia que deberá trasmitir a los clientes. Resultando de alta aplicabilidad y de posible generalización, desde su aspecto metodológico, a otras instalaciones con características similares; radicando en ello su novedad científica y práctica. 


\section{Referencias Bibliográficas:}

Arbelo, M. (2015). Diseño del producto turístico "Viviendo en la campiña" en la CCS Juan Verdecia de Camajuaní. Tesis en opción al Título de Máster en Gestión Turística. Las Villas, Cuba: Facultad de Ingeniería Industrial y Turismo. Universidad Central Marta Abreu.

Baena Paz, G. (2014). Metodología de la Investigación. México, Grupo Editorial Patria.

Bulnes, DM. (2015). Diseño preliminar del Plan de Desarrollo Turístico. Municipios Seleccionados de la provincia Mayabeque para el período 2015-2020.Tesis en opción al Título de Máster en Gestión Turística. La Habana, Cuba. Facultad de Turismo. Universidad de La Habana.

Castro, U, González, J.A y. Maldonado L.M. (2017). Destinos turísticos inteligentes: ¿Estrategia para el desarrollo local en países pobres? En, Rev. Revista Turydes. Turismo y Desarrollo. N. 22, pp 15-18.

Cisneros Mustelier, L (2010). Teoría y Técnica del Turismo. Cuba. Facultad de Turismo. Universidad de La Habana.

Cuba, Anuario Estadístico (2016).

Cuba, Partido Comunista de Cuba (2016). Actualización de los Lineamientos de la política económica y social del Partido y la Revolución para el período 2016-2021.

Hernández Sampieri, R. y otros. (2014). Metodología de la Investigación. México, Ed. McGraw-Hill / Interamericana editores, S.A. de C.V.

Perelló, J. L. (2001). Desarrollo y Promoción de Productos Turísticos. Universidad de La Habana, Facultad de Turismo, La Habana. Cuba.

Perelló, J. L. (2015). Actualización del Turismo en Cuba. Documento de Trabajo DT3/15. Facultad de Turismo, Universidad de La Habana.

Únger Pérez, R (2016). La Isla de los Pinos Nuevo. Cuba, Editorial El Abra.

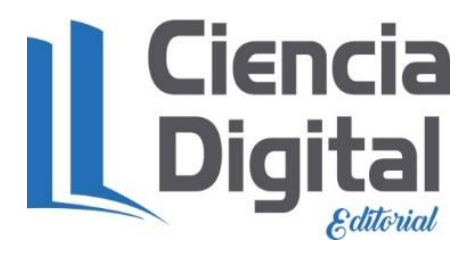




\section{PARA CITAR EL ARTÍCULO INDEXADO.}

Arias Pérez, L., González Curbelo, W., \& Cisneros Mustelier, L. (2020). Ruta histórica cultural "el abra": un producto turístico enfocado al turismo interno en la isla de la juventud. Explorador $\quad$ Digital, $\quad 4(3), \quad 18-31$. https://doi.org/10.33262/exploradordigital.v4i3.1277

\section{Ciencia Ligital}

El artículo que se publica es de exclusiva responsabilidad de los autores y no necesariamente reflejan el pensamiento de la Revista Explorador Digital.

El artículo queda en propiedad de la revista y, por tanto, su publicación parcial y/o total en otro medio tiene que ser autorizado por el director de la Revista Explorador Digital.
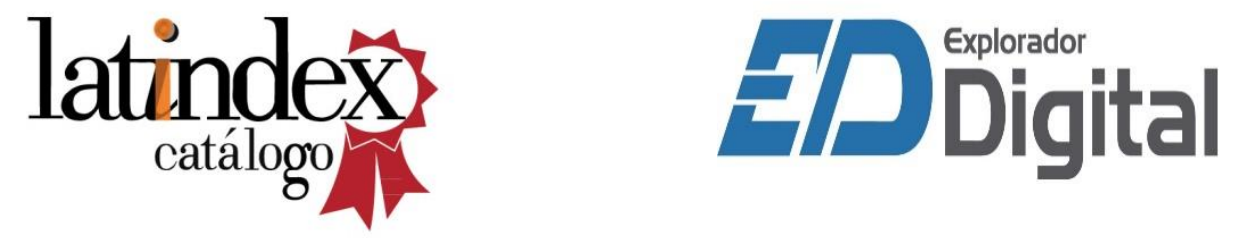